\title{
A Field Study to Collect Expert Knowledge for the Development of AR HUD Navigation Concepts
}

\author{
Matthias Schneider \\ Media Informatics Group, \\ University of Regensburg, GER \\ Daimler AG, Stuttgart, GER \\ Matthias.sc.schneider@daimler.com

\section{Anna Bruder} \\ Daimler AG, Stuttgart, GER \\ Anna.bruder@daimler.com \\ Marc Necke \\ Daimler AG, Stuttgart, GER \\ Marc.necker@daimler.com \\ Tim Schluesener \\ Daimler AG, Stuttgart, GER \\ Tim.schluesener@daimler.com

\section{Niels Henze} \\ Media Informatics Group, \\ University of Regensburg, GER \\ Niels.henze@ur.de

\section{Christian Wolff} \\ Media Informatics Group, \\ University of Regensburg, GER \\ Christian.wolff@ur.de
}

Permission to make digital or hard copies of part or all of this work for personal or classroom use is granted without fee provided that copies are not made or distributed for profit or commercial advantage and that copies bear this notice and the full citation on the first page. Copyrights for third-party components of this work must be honored. For all other uses, contact the Owner/Author.

AutomotiveUI '19 Adjunct, September 21-25, 2019, Utrecht, Netherlands (c) 2019 Copyright is held by the owner/author(s).

ACM ISBN 978-1-4503-6920-6/19/09.

https://doi.org/10.1145/3349263.3351339

\begin{abstract}
A promising advancement of conventional head-up displays in vehicles is the implementation of augmented reality. By projecting the content onto the vehicle's windshield, information can be displayed in a contact analogue way in the real world. Two major challenges for concept developers are to reduce masking caused by augmented reality content and to create concepts that are suitable for the limited field of view. To approach these challenges, we designed two contact analogue navigation concepts and evaluated them in a field study with a prototype car that contained a complete AR HUD testing environment. The subjects were experts in interaction design, AR, HUD and sales. First results of the experts' suggestions for improvements are given in this extended abstract.
\end{abstract}

\section{Author Keywords}

Augmented reality; head-up display; field study; contact analogue; navigation; masking

\section{CCS Concepts}

- Human-centered computing Field studies • Humancentered computing Mixed / augmented reality 


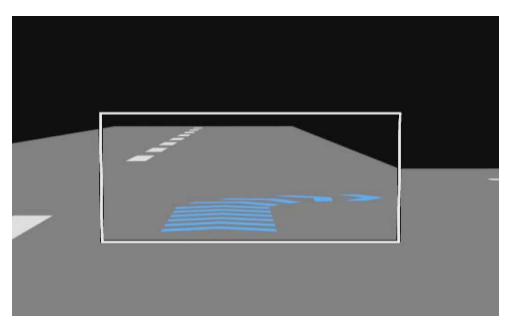

Figure 1: The entry marker of the solid fishbone concept.

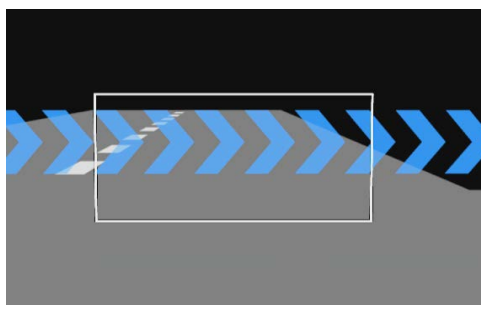

Figure 2: The middle marker of the solid fishbone concept.

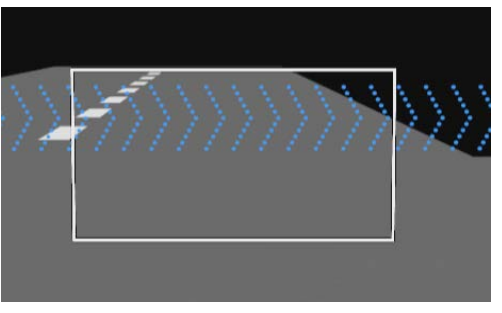

Figure 3: The middle marker of the dotted fishbone concept.

\section{Introduction}

Today's cars are equipped with a large number of driving assistance systems (DAS) to support the driver in challenging traffic situations [2]. One example for current DAS technologies are conventional head-up displays (HUDs). This technology makes it possible to display driving-related information in the driver's primary field of view (FOV) through a virtual image that is mirrored in the windshield. The next step in the development process of HUDs is the integration of augmented reality (AR) [1]. This allows the combination of virtual and real information in the driver's primary FOV and is called augmented reality head-up display (AR HUD). This was realized in a car for the first time by Bubb in 1975 [4]. The AR HUD is supposed to support drivers through a more intuitive way of displaying driving assistance information [3]. There are still several challenges to master before an AR HUD can be realized in a production vehicle. One of those is the correct superimposition of virtual information onto the real environment. Another challenge is that, due to technical limitations, AR content can only be displayed in a small area of the windshield. Therefore, it is necessary to design for more complex traffic situations, such as curves, because the virtual information might be cut off. Additionally, superimposing virtual information onto the real traffic environment can lead to driver distraction, annoyance, and masking of other road users [10]. Thus, a further challenge in concept development for AR HUD is to reduce masking effects while ensuring that the same level of information is provided.

In this field study, two contact analogue navigation concepts for AR HUD are compared in terms of their degree of masking the real environment and how appropriate they are for the use case navigation. The goal is to examine whether masking effects can be reduced while maintaining a consistent interpretability of the navigation cues by the integration of Gestalt Principles. Furthermore, we want to collect expert knowledge to derive guidelines for AR HUD navigation concept design. This experiment is one of the first to utilise a prototype car with a complete AR HUD environment that includes real sensor and road map data.

\section{Navigation Concept Design}

Based on guidelines from research, especially from Pfannmüller [10] and Israel [6], and focus groups with experts in the field of AR HUD concept design, two navigation concepts for AR HUD have been created. For both concepts a light blue colour was chosen: RGB $(102,153$, 230) with a transparency of 0.

\section{The Solid Fishbone Concept}

The solid fishbone concept consists of two basic elements, entry marker and middle marker. Both components are in the shape of a fishbone as recommended by e.g. Israel [6], to result in a smoother cut when leaving the FOV as well as less masking of the real world. The entry marker consists of a curved fishbone that virtually lies on the street in a contact analogue way while showing the upcoming turn (see Figure 1). When the driver approaches a manoeuvre point, the entry marker fades in 90 meters in advance with an initial slanted position (tilt $=15^{\circ}$ ) to achieve a better visibility. While getting closer to the manoeuvre, the tilt is reduced to $0^{\circ}$ in a steady manner and the entry marker fades out. Next, the middle marker fades in resembling a wall of upright fishbones that is placed in the centre of the road the upcoming manoeuvre points into (see Figure 2). The middle marker is longer than the FOV and is also shown during turning. The fade in and out time is 0.5 seconds for both of the elements. 


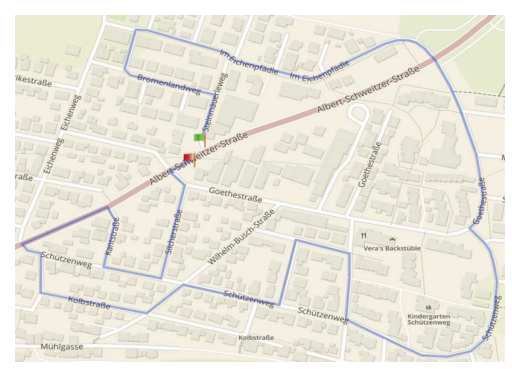

Figure 4: The track had a length of 2.5 $\mathrm{km}$ and contained nine right as well as nine left turns and one straight manoeuvre crossing a street. Also, a little roundabout was on the test track.

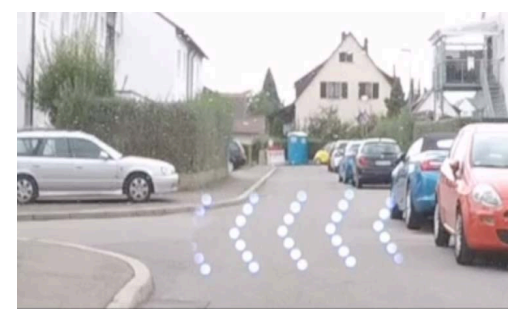

Figure 5: Interface of the test vehicle.

\begin{tabular}{cl} 
Votes & Positive feedback \\
\hline 7 & $\begin{array}{l}\text { notifications are pleasant in } \\
\text { terms of their colour scheme }\end{array}$ \\
5 & $\begin{array}{l}\text { contact analogue positioning } \\
5\end{array}$ \\
3 & $\begin{array}{l}\text { fade-in timing of the fishbones } \\
\text { ereasing }\end{array}$
\end{tabular}

Table 1: Positive feedback related to both concept variants.
The Dotted Fishbone Concept

The components and their behaviour are the same for both of the navigation concepts with the only difference that the core element of the dotted fishbone concept is a fishbone consisting of dots (see Figure 3 ). The idea behind that is that the driver should still recognize the fishbone shape with the help of Gestalt Principles, especially Principle of Proximity and Principle of Good Continuation. At the same time, the dotted design should lead to a lower degree of masking. However, it might happen that the mental workload of the driver is higher while using the dotted fishbones because its elements might be harder to recognize and interpret than the solid ones [8].

\section{Experiment}

In this section information about study participants, the test track, the test environment, and the procedure is given.

\section{Participants}

To get valuable expert knowledge about masking effects and the development of future navigation concepts, only experts in fields relevant for the development of an AR HUD were chosen as subjects. In total, 26 participants took part in the study. Two datasets had to be excluded from the analysis due to technical problems. Of the remaining 24 participants, five were female and 19 male, ages ranging from 22 to 51 years. Every driver had used a static HUD before but only one participant had one in his private car. The participants' attitude toward HUD in general was positive. All subjects gave written consent to participate in the study.
Test Track

An important factor for the test track was a consistent traffic density during the drives were conducted (between 9:00 am and 4:00 pm). The chosen test track (see Figure 4) mainly consisted of streets with a speed limit of $30 \mathrm{kph}$. With the help of predefined waypoints, the test vehicle showed the manoeuvres of the test track even though a free navigation or redirection would have been possible with this system as well.

\section{Test Environment}

As the test vehicle, a prototype with integrated AR HUD was used. Figure 5 shows an image of the interface of the prototype car which shows the middle marker of the dotted fishbones. Furthermore, a predevelopment environment software was created for the car. It includes the so-called Robot Operating System (ROS) that is used to synchronize the vehicle's sensor and bus data (GPS, accelerometer, gyroscope, and FlexRay). Also, a map interface by HERE maps was included to access current map data online. With the help of the toolkit $Q T$, a graphical user interface for AR HUD was created and additionally, the Open Graphics Library (Open GL) was used as rendering pipeline. For the AR content creation, the 3D tool Autodesk Maya was used.

\section{Procedure}

After getting an introduction to the test vehicle, the participants followed a short practice route to familiarise themselves with the test vehicle. Next, the subjects stopped at a parking area next to the starting point of the track. The HUD was calibrated along the y-axis with the help of a fixed point. While the examiner started the test environment, the drivers filled out a questionnaire covering demographical data, driving experience, experience with HUD, and the weather conditions. Next, the 


\begin{tabular}{cl} 
Votes & Negative feedback \\
\hline 18 & $\begin{array}{l}\text { imprecise positioning of the nav- } \\
\text { igation cues }\end{array}$ \\
7 & $\begin{array}{l}\text { transition between entry and } \\
\text { middle marker is too abrupt }\end{array}$ \\
6 & $\begin{array}{l}\text { entry marker should be dis- } \\
\text { played longer }\end{array}$ \\
3 & Entry marker too small \\
2 & Entry marker appears too late
\end{tabular}

Table 2: Negative feedback which is related to both concept variants.

\section{Votes Suggestions for improvements}

9|15 Include possibility to adjust concept concerning size, number of elements, and colour

$7 / 9$ Improve positioning in general

$6 / 4$ Integration of pre-indication

212 Integrate permanent notification to show that system is running

$3 \mid 1 \quad$ Extend display time of entry marker

2|0 Make brightness adjustable

Table 3: Suggestions for improvements which are related to both concept variants. The votes on the left side have been given for the solid fishbones, the votes on the right side for the dotted ones. subjects were asked to think aloud during the drives. Every participant completed the test track twice with identical test procedures for both AR HUD concepts. The order in which the concept variants were tested was counterbalanced across participants to mitigate the influence of training effects on the performance measures used. The examiner was seated next to the driver while a second person was sitting in the back to log special incidents like navigational errors. Additionally, a GoPro dashcam was integrated in the test vehicle to record audio and video data. If subjects were insecure about interpreting the navigational cues, they were asked to tell the examiner in which direction the navigational cue pointed. This was done to avoid possible rerouting inconsistencies. After finishing the route, participants filled out the User Experience Questionnaire (UEQ) [5] and the Driving Activity Load Index (DALI), a tool designed to evaluate the driver's mental workload [9]. In addition, a self-designed questionnaire based on Pfannmüller [10] to assess design, degree of masking, distraction, and positioning accuracy was used. An open questions part was included to find out what the drivers liked or disliked about the navigation concepts and what they would improve. After the second drive, the subjects were asked to compare the concepts, state their favourite and to explain their decision. Also, they stated ideas on how to combine static and contact analogue elements.

\section{First Results}

First results of the open questions asked are given in this section. The evaluation was conducted according to the qualitative content analysis by Mayring et al. [7]. There was positive and negative feedback that was valid for both concepts which is listed in Table 1 and 2 respectively. In addition, the subjects made suggestions for improvements concerning both concepts (see Table 3 ).
When asked which concept they preferred and for what main reasons, the results showed a slight preference for the solid fishbones. In total, the solid fishbones got 12 votes, the dotted fishbones got nine, and three experts had no favourite. In case of the solid fishbone concept, six subjects mentioned that it is easier to interpret, especially when the AR content is cut off strongly due to the limited FOV and only parts of the fishbones remain visible. Three participants thought the solid fishbones had a better design. For the dotted fishbone concept, six participants mentioned less masking as one of their main reason for preferring it. Two experts said that their cognitive load was lower and also, the design was preferred two times.

\section{Discussion and Conclusion}

In this study, an open questions part was included to collect expert knowledge in terms of navigation concepts for AR HUD which can be used for future concept development. First results have been given already and further findings can be looked up in Schneider et al. [11] together with the results of the evaluated masking effects. Even though the concepts were only evaluated in one specific car, this study can be seen as one of the first approaches to investigate navigation concepts for an AR HUD in the real traffic. We used experts in the field of AR HUD development as subjects to get detailed information on what a suitable navigation concept for AR HUD should look like and on how critical the caused masking effects are. Future studies should also focus on participants without expert knowledge to transfer the results to the general population. Also, masking effects could be examined further by integrating objective measurements like physical targets placed on a private test track. 


\section{References}

1. Ronald T. Azuma. 1997. A Survey of Augmented Reality. Teleoperators and Virtual Environments 6, 4: 355-385.

https://doi.org/10.1162/pres.1997.6.4.355

2. Klaus Bengler, Klaus Dietmayer, Berthold Faerber, Markus Maurer, Christoph Stiller, and Hermann Winner. 2014. Three Decades of Driver Assistance Systems: Review and Future Perspectives. IEEE Intelligent Transportation Systems Magazine 6, 4: 622. https://doi.org/10.1109/MITS.2014.2336271

3. Klaus Bengler, Martin Götze, Lisa Pfannmüller, and Albert Zaindl. 2015. To See or not to See - Innovative Display Technologies as Enablers for Ergonomic Cockpit Concepts. Ergonomic Requirements, Future Mobility, Future Functionality. In Proceedings of the electronic displays Conference.

4. Heiner Bubb. 1975. Untersuchungen über die Anzeige des Bremsweges im Fahrzeug. Doctoral Dissertation. Technical University of Munich, Munich, GER

5. Andreas Hinderks, Martin Schrepp, and Joerg Thomaschewski. 2018. UEQ - User Experience Questionnaire. Retrieved September 12, 2018 from http://www.ueq-online.org/

6. Boris Israel. 2013. Potenziale eines kontaktanalogen Head-up Displays für den Serieneinsatz. Doctoral Dissertation. Technical University of Munich, Munich, GER.

7. Philipp Mayring and Thomas Fenzl. 2014. Qualitative Inhaltsanalyse. In Handbuch Methoden der empirischen Sozialforschung, Nina Baur and Joerg Blasius (eds.). Springer VS, Wiesbaden, GER, 543556. https://doi.org/10.1007/978-3-531-189390_38

8. Wolfgang Narzt, Gustav Pomberger, Alois Ferscha, Dieter Kolb, Reiner Mueller, Jan Wieghardt, Horst Hoertner, and Christopher Lindinger. 2006. Aug- mented reality navigation systems. Universal Access in the Information Society 4, 3: 177-187. https://doi.org/10.1007/s10209-005-0017-5

9. Annie Pauzié. 2008. A method to assess the driver mental workload: The driving activity load index (DALI). IET Intelligent Transport Systems 2, 4: 315-322. https://doi.org/10.1049/iet-its:20080023

10. Lisa Pfannmüller. 2017. Anzeigekonzepte für ein kontaktanaloges Head-up Display. Doctoral dissertation. Technical University of Munich, Munich, GER.

11. Matthias Schneider, Anna Bruder, Marc Necker, Tim Schluesener, Niels Henze, and Christian Wolff. 2019. A real-world driving experiment to collect expert knowledge for the design of AR HUD navigation that covers less. In Mensch und Computer 2019 - Workshopband, Wolf and Steinicke (eds.). Bonn: Gesellschaft für Informatik e.V.. 